\title{
基于TELEMAC-2D的不同设计暴雨下厦门岛城市 内涝特征分析
}

\author{
刘家宏 ${ }^{1,3^{*}}$, 李泽锦 ${ }^{1}$, 梅超 $^{1}$, 王开博 ${ }^{1}$, 周冠南 ${ }^{2}$ \\ 1. 中国水利水电科学研究院, 流域水循环模拟与调控国家重点实验室, 北京 100038 ; \\ 2. 中国铁建大桥工程局集团有限公司, 天津 300300; \\ 3. 水利部水资源与水生态工程技术研究中心, 北京 100044 \\ * 联系人, E-mail: liujh@iwhr.com
}

2018-11-29 收稿, 2018-02-25 修回, 2019-02-28 接受, 2019-07-03 网络版发表

国家自然科学基金(51739011)和国家重点研发计划(2016YFC0401401，2018YFC1508203)资助

\begin{abstract}
摘要 随着城市化率的提高, 近年来我国城市内涝灾害频发. 数值模拟是城市内涝研究的重要手段, 已广泛应用于 城市内涝特征分析. 本文以厦门岛为研究区，建立了基于TELEMAC-2D的城市内涝模型，模拟了不同重现期(50, $100 \mathrm{a})$ 和不同雨峰系数 $(0.2,0.3,0.4,0.5,0.6,0.7,0.8)$ 下厦门岛内涝积水情况. 结果表明: 积水总量受雨峰系数的影 响较大, 雨峰系数越大, 积水总量的峰值越大; 积水面积的变化受雨峰系数的影响, 在同一重现期下, 积水面积随雨 峰系数的增大而增大; 不同内涝风险等级下的积水面积受雨峰系数的影响是不同的, 积水深度大于 $15 \mathrm{~cm}$ 的积水面 积随雨峰系数的增大而增大, 而积水深度大于 $30 \mathrm{~cm}$ 的积水面积变化受雨峰系数的影响不明显; 在同样的重现期下, 雨峰系数越大, 迟滞时间越短.同时, 对于城市排水管网的简化方法进行了相应的讨论和分析.
\end{abstract}

关键词 城市内涝, TELEMAC-2D水动力学模型, 积水模拟, 城市化, 厦门岛

在全球气候变化的大背景下，随着我国城市化进 程地加速推进, 我国城市内涝灾害发生频率增加、灾 害损失加剧, 已成为影响经济社会发展的一种主要自 然灾害 ${ }^{[1 \sim 3]}$. 住建部门统计表明, 2008 2010年，全国有 213 个城市发生过不同程度的内涝, 其中内涝灾害超过 3 次以上的城市有 137 个, 57 个城市的最长积水时间超 过 $12 \mathrm{~h}^{[4]}$, 近几年来, 北京 ${ }^{[5]} 、$ 武汉 ${ }^{[6]} 、$ 深圳 ${ }^{[7]}$ 、厦门 ${ }^{[8]}$ 等城市更是因城市内涝引起重大损失, 城市内涝灾害 日益受到社会各界的广泛关注 ${ }^{[9 \sim 12]}$. 据联合国有关数 据表明，当前有 $55 \%$ 的人口居住在城市地区，而这一比 例在 2050 年左右将会上升到 $68 \%{ }^{[13]}$. 我国2017年城市 化率已达 $58.52 \%{ }^{[14]}$, 在新型城镇化背景的影响下, 未 来我国城市化进程将进一步加快, 城市内涝灾害研究 的重要性也愈加凸显. 城市内涝情景模拟是分析城市
内涝灾害特征的重要方法, 通过不同暴雨情景下的城 市内涝数值模拟, 分析城市的积水特征, 对于科学评估 城市内涝的产生特征、研究其演化机制和预防对策具 有重要的实践意义 ${ }^{[15 ~ 18]}$. 厦门岛地处东南沿海, 受台风 暴雨影响，城市内涝灾害严重，如2016年“莫兰蒂”和 2018年“山竹”两次台风暴雨均造成厦门岛严重积水, 影响城市正常运转 ${ }^{[19]}$. 本文针对岛屿型城市, 以厦门岛 为例开展基于数值模拟的不同设计暴雨下的城市内涝 特征分析研究.

\section{1 研究数据}

\section{1 研究区概况}

本文的研究区为中国福建省厦门市的厦门岛. 厦 
门岛坐落于我国东南沿海区域, 福建九龙江人海处, 属 于亚热带海洋性气候, 多年平均降雨量为 $1513.3 \mathrm{~mm}$ (1965 2015年), 地势南高北低, 面积约为 $142.9 \mathrm{~km}^{2}$, 研 究区位见图1所示. 厦门岛是典型的岛屿型城市, 降水 时空差异较大, 年内年际分配不均, 年内降雨主要集中 在5 8月. 厦门岛的城市化率超过 $80 \%$, 下垫面不透水 率较高, 排涝水系较少且大多数已渠道化、暗涵化, 每 年的雨季极易产生城市内涝灾害. 厦门岛四周环海, 降 雨除了汇入排水管网以外, 主要是依地势就近人海, 可 视作一个完全独立的汇水区. 由于厦门岛的城市化率 比较高, 排水系统独立, 并且关于岛屿型城市的内涝问 题研究相对较少, 因此, 以厦门岛为例研究岛屿型城市 在不同设计暴雨条件下的内涝特征具有一定的典型 意义.

\section{2 数据来源及处理}

\subsection{1 设计暴雨数据}

根据福建省气候中心在2016年修订的《厦门市暴 雨强度公式修编和设计雨型研究技术报告》, 并考虑 到对城市内涝危害最大的是短历时强降雨, 采用短历 时暴雨强度公式生成设计暴雨情景, 设计暴雨强度公 式如下 ${ }^{[20]}$ :

$q=\frac{928.15 \times(1+0.716 \lg P)}{(t+4.4)^{0.535}}$,

式中, $q$ 是暴雨强度 $\left(\mathrm{L} /\left(\mathrm{s} \mathrm{hm}^{2}\right)\right), P$ 是重现期(a), $t$ 是降雨 历时 (min). 在本文的设计暴雨中, 以雨峰系数 $r$ 代表不 同雨型的峰现时间占总降雨历时的比例, 设计了 2 种重 现期 $(50,100 \mathrm{a})$ 和 7 种雨峰系数 $(0.2,0.3,0.4,0.5,0.6$, $0.7,0.8$ )共 14 场短历时设计暴雨, 历时均为 $120 \mathrm{~min}$.

本文采用二维水动力学模型进行城市内涝淹没模 拟. 根据《GB50014-2006(2014版)室外排水设计规 范》和有关研究 ${ }^{[8,21]}$, 结合厦门岛现场调研情况设定, 厦门岛城市排水管网实际排涝能力为一年一遇, 即根 据暴雨强度公式计算可得, 厦门市一年一遇的历时 $120 \mathrm{~min}$ 降雨的平均雨强为 $0.425 \mathrm{~mm} / \mathrm{min}$. 在降雨数据 中将小于 $0.425 \mathrm{~mm} / \mathrm{min}$ 的部分扣除, 视为通过排水管 网排走, 雨强大于 $0.425 \mathrm{~mm} / \mathrm{min}$ 的部分落到地面形成 地表径流 ${ }^{[22]}$. 综合降雨和排水管网的排水作用, 14场设 计暴雨的设计降雨过程见图2所示.

\subsection{2 厦门城市下垫面数据}

土地利用类型是通过卫星遥感影像数据解译得到 (图3). GF-1号卫星遥感数据可以从“中国卫星资源应用

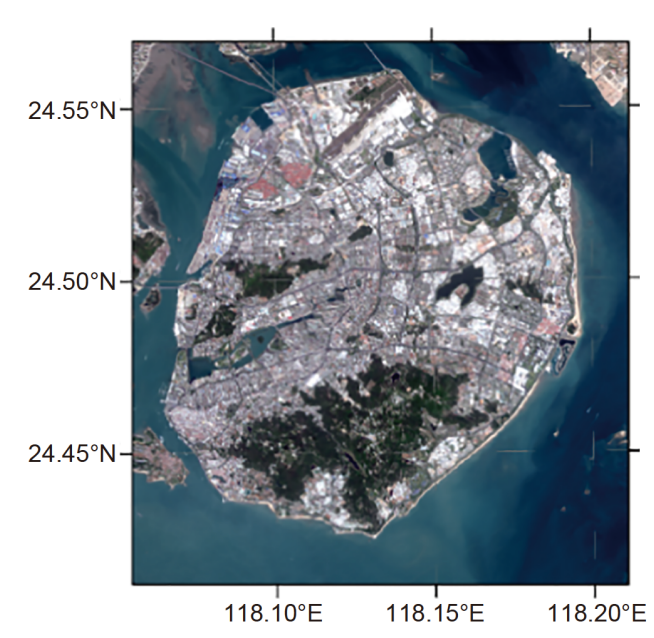

图 1 厦门岛研究区位图

Figure 1 The location of Xiamen Island

中心”(http://www.cresda.com/CN/)下载获取, 分辨率为 $16 \mathrm{~m}$. 根据研究目的将厦门岛的土地利用类型划分为 5 种类型: 绿地、道路、水面、建筑和其他. 并且将建筑 物的地理高程统一加高 $10 \mathrm{~m}$, 道路的地理高程统一加 高 $0.15 \mathrm{~m}$.

土壤类型的数据可以从“寒区旱区科学数据中心” 网站(http://westdc.westgis.ac.cn/data/)下载获取. 并根据 美国SCS模型的土壤分类标准 ${ }^{[23]}$, 厦门岛的土壤分组 属于 $\mathrm{B}, \mathrm{C}, \mathrm{D}$ 三种, 其中 $\mathrm{B}$ 类土表示透水性较强, $\mathrm{C}$ 类土 表示透水性较弱, D类土表示透水性很弱.

模型采用的DEM高程数据可以从 “地理空间数据 云”网站(http://www.gscloud.cn/)下载获取, 分辨率是 $30 \mathrm{~m} \times 30 \mathrm{~m}$. 参考以往研究成果, $30 \mathrm{~m}$ 的分辨率可以整 体上反映出研究区的坡度、地形变化等特征, 但也存 在一定的不确定性, 通过ArcGIS的水文分析工具对 DEM数据进行分析处理, 对于不同设计降雨情况下的 城市内涝特征的研究基本可以满足要求.

曼宁系数的确定是综合考虑了土地利用类型和水 文土壤分组的结果, 并参考研究区域与本研究区域位 置相近、气候相似的文献[24]. 根据土地利用类型的不 同, 将绿地、道路、水面、建筑面和其他的曼宁系数 分别设置为 $0.08,0.05,0.015,0.2$ 和 0.05 .

\section{2 研究方法}

\section{1 二维水动力学城市内涝模型}

本文采用TELEMAC-2D模型进行城市内涝的二维 水动力学模拟. TELEMAC-MASCARET模型是法国国 

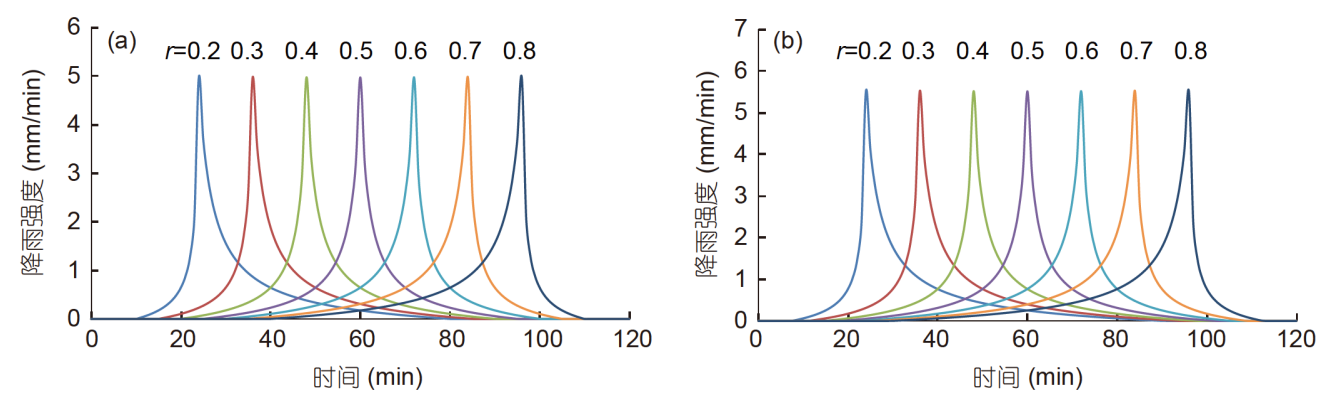

图 2 不同重现期和不同雨峰系数的设计降雨过程. (a) 50年一遇; (b) 100 年一遇

Figure 2 Design hyetograph with different return periods and different coefficients for the storm peak. (a) 50-year return period; (b) 100-year return period

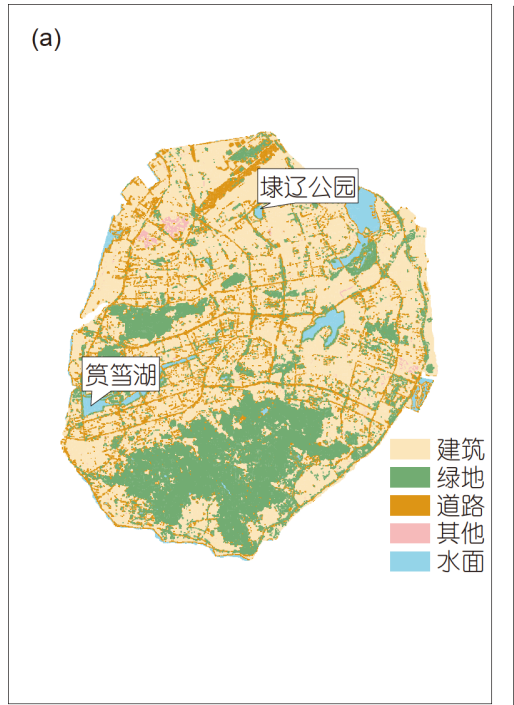

(b)

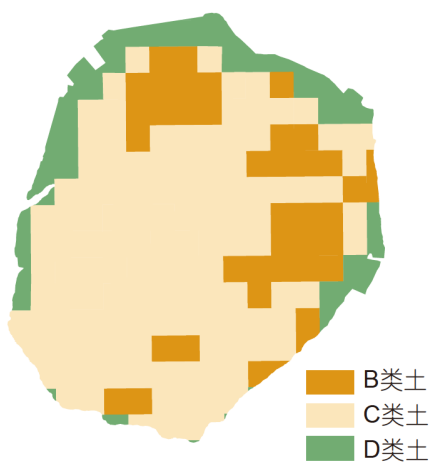

(c)

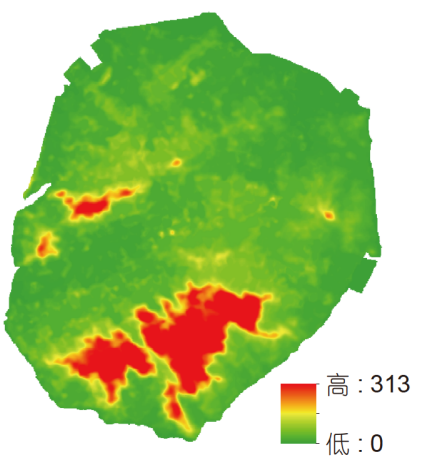

图 3 厦门岛城市下垫面数据. (a) 土地利用类型; (b) 水文土壤分组; (c) DEM高程

Figure 3 The underlying surface in Xiamen Island. (a) Landuse type; (b) soil classification; (c) DEM data

家水力学与环境实验室(National Hydraulic and Environment Laboratory)开发的开源的河流、河口和海岸的 一维、二维和三维水力学模型系统. 利用有限元数值 方法求解Navier-Stokes方程, 并在V6P1和V6P2版本中 新添加了有限体积法. 本次模拟使用的模型版本是 V7P2版本，采用非结构网格进行计算域剖分. TELEMAC-2D是TELEMAC-MASCARET系列中的一个二 维模块, 可以用来求解二维圣维南方程, 模拟计算二维 自由表面流，在水动力学模拟和海岸风暴潮模拟中得 到了广泛应用 ${ }^{[25 ~ 27]}$.

(i ) 模型基本方程. 二维浅水方程的非守恒形式 如下(不考虑科氏力和风阻):

$\frac{\partial h}{\partial t}+\frac{\partial(h u)}{\partial x}+\frac{\partial(h v)}{\partial y}=0$, $\frac{\partial u}{\partial t}+u \frac{\partial u}{\partial x}+v \frac{\partial u}{\partial y}=-g \frac{\partial Z}{\partial x}+F_{x}+\frac{1}{h} \operatorname{div}\left(h v_{\mathrm{e}} \nabla u\right)$,

$\frac{\partial v}{\partial t}+u \frac{\partial v}{\partial x}+v \frac{\partial v}{\partial y}=-g \frac{\partial Z}{\partial y}+F_{y}+\frac{1}{h} \operatorname{div}\left(h v_{\mathrm{e}} \nabla v\right)$,

其中 $u, v$ 分别是 $x$ 和 $y$ 方向的流速, $t$ 是时间, $h$ 是水深, $Z$ 是 自由水位高程, $F_{x}, F_{y}$ 分别是 $x$ 和 $y$ 方向的阻力分量, $v_{\mathrm{e}}$ 是 有效黏性系数, $g$ 是重力加速度, div表示散度(divergence)计算符号, $\nabla$ 是梯度运算符号.

TELEMAC-2D采用非守恒形式的二维浅水动力方 程, 将水深和流速作为变量, 采用基于特征线方法的分 步算法. 在第一个时间步, 根据方程组的双曲性, 对与 $h$, $u, v$ 相关的对流项进行处理; 在第二个时间步处理剩余 的各项, 包括传播项、扩散项、源项等, 将方程各项进 行线性化, 采用有限元方法进行离散求解. 
分步方法如下 ( $f$ 代表 $h, u, v$ 的函数):

$\frac{\widetilde{f}-f^{n}}{\Delta t}+$ 对流项 $=0$,

$\frac{f^{n+1}-\widetilde{f}}{\Delta t}+$ 其他 $=0$.

(ii) 时间项的离散.

$\frac{\partial f}{\partial t}=\frac{f^{n+1}-f^{n}}{\Delta t}$.

采用差分方法进行离散，上标 $n$ 代表第 $n$ 个时间步, 即: $f^{n}=f^{0}+n \Delta t$, 式中, $f$ 代表 $h, u, v$ 的函数. 底摩阻项 采用全隐式处理, 其余项采用半隐式处理.

经过离散后的方程组为 $\left(\theta_{\mathrm{h}}\right.$ 为水深的隐式差分系数):

$$
\begin{aligned}
& \frac{h^{n+1}-h^{n}}{\Delta t}+\vec{u}_{\mathrm{conv}} \cdot \nabla h+h_{\mathrm{prop}} \operatorname{div}(\vec{u})=0, \\
& \frac{u^{n+1}-u^{n}}{\Delta t}+\vec{u}_{\mathrm{conv}} \cdot \nabla u \\
= & -\theta_{\mathrm{h}} g \frac{\partial\left(h^{n+1}-h^{n}\right)}{\partial x}-g \frac{\partial Z^{n}}{\partial x}+F_{x}+\frac{1}{h} \operatorname{div}\left(h v_{\mathrm{e}} \nabla u^{n+1}\right), \\
& \frac{v^{n+1}-v^{n}}{\Delta t}+\vec{u}_{\mathrm{conv}} \cdot \nabla v \\
= & -\theta_{\mathrm{h}} g \frac{\partial\left(h^{n+1}-h^{n}\right)}{\partial y}-g \frac{\partial Z^{n}}{\partial y}+F_{y}+\frac{1}{h} \operatorname{div}\left(h v_{\mathrm{e}} \nabla v^{n+1}\right) .
\end{aligned}
$$

(iii) 空间项的离散. TELEMAC-2D采用有限元方 法进行方程的离散. 将函数在空间离散的节点上进行 分解, 即: $f=\sum_{i=1}^{n} f_{i} \psi_{i}$, 其中 $n$ 是离散点的数量, $f_{i}$ 是函数 $f$ 在 $i$ 点的值, $\psi_{i}$ 是一组与节点相关的线性无关的基函数. TELEMAC-2D采用的对水深的基函数和对速度的基函 数是不同的.

对时间离散后的方程组用变分公式进行计算，并 在计算域上积分，对得到的方程组进行分部积分计算, 用基函数取代相应的 $h, u, v$ 函数, $n_{p h}, n_{p u}, n_{p v}$ 分别为对 应的离散点数量:

$$
h=\sum_{i=1}^{n_{p h}} h_{i} \psi_{i}^{\mathrm{h}}, \quad u=\sum_{i=1}^{n_{p u}} u_{i} \psi_{i}^{\mathrm{u}}, \quad v=\sum_{i=1}^{n_{p v}} v_{i} \psi_{i}^{\mathrm{v}},
$$

\section{经过一系列整理分析得到模型最终方程 ${ }^{[28]}$.}

\section{2 模型降雨产流模拟方法}

TELEMAC-2D的下渗模型采用的是美国的SCSCN方法. SCS-CN方法是1954年由美国Soil Conservation Service部门根据不同地区小流域降雨径流资料的 研究得出的一个经验性模型, 采用Curve Number $(\mathrm{CN})$
系数来描述下渗能力. $\mathrm{CN}$ 值的主要影响因素是水文土 壤分组、土地利用方式、水文地形条件和前期土壤条 件(AMC)等. SCS方法的公式如下 ${ }^{[29,30]}$.

$S=25.4 \times\left(\frac{1000}{C N}-10\right)$,

其中 $S$ 是潜在的土壤最大蓄水量.

为提高参数 $\mathrm{CN}$ 值的合理性，通过查阅美国土壤保 护局TR-55手册 $\mathrm{CN}$ 值表 ${ }^{[23]}$ 和参考研究区域与本研究区 域水文地形条件相似的相关文献 $[31,32]$, 结合土地利 用类型和水文土壤分组结果，考虑到厦门岛地处东南 沿海, 气候湿润, 前期土壤条件为AMC-II. 因此, 在本 次模拟中 CN值按表1进行确定.

\section{3 模型构建}

采用TELEMAC-2D模型进行数值模拟分析，利用 BlueKenue软件将研究区域划分为三角形网格, 共计 174310 个节点, 346862 个网格, 分辨率是 $30 \mathrm{~m}$. 模型设 置的边界条件是自由出流边界条件, 初始地表无积水 降雨, 前期土壤含水量按照SCS-CN方法设为AMC-II, 即中等情况. 降雨持续 $120 \mathrm{~min}$, 模拟时长 $360 \mathrm{~min}$, 时 间步长 $0.5 \mathrm{~s}$, 库朗数 $(\mathrm{CFL})$ 设为 0.75 . 在对排水管网进行 科学概化的基础上建立厦门岛的二维水动力学模型, 在不同设计暴雨条件下, 模拟厦门岛城市内涝过程.

\section{3 结果分析}

\section{1 不同设计暴雨情景下的积水总量特征分析}

图 4 为重现期为 100 年条件下，不同雨峰系数下积 水总量达到峰值的时刻的积水内涝分布图. 对模拟结 果进行适当的统计分析，得到各场设计暴雨情况下积 水总量随时间变化的过程图，见图5; 并得到各场设计

表 1 厦门岛不同土地利用类型和水文土壤分组的 $\mathrm{CN}$ 值

Table 1 The $\mathrm{CN}$ values of Xiamen Island with different landuse type and soil classification

\begin{tabular}{cccc}
\hline CN值 & B类土 & C类土 & D类土 \\
\hline 绿地 & 57 & 71 & 80 \\
道路 & 92 & 94 & 98 \\
水面 & 98 & 100 & 100 \\
建筑面 & 94 & 96 & 98 \\
其他 & 80 & 88 & 92 \\
\hline
\end{tabular}



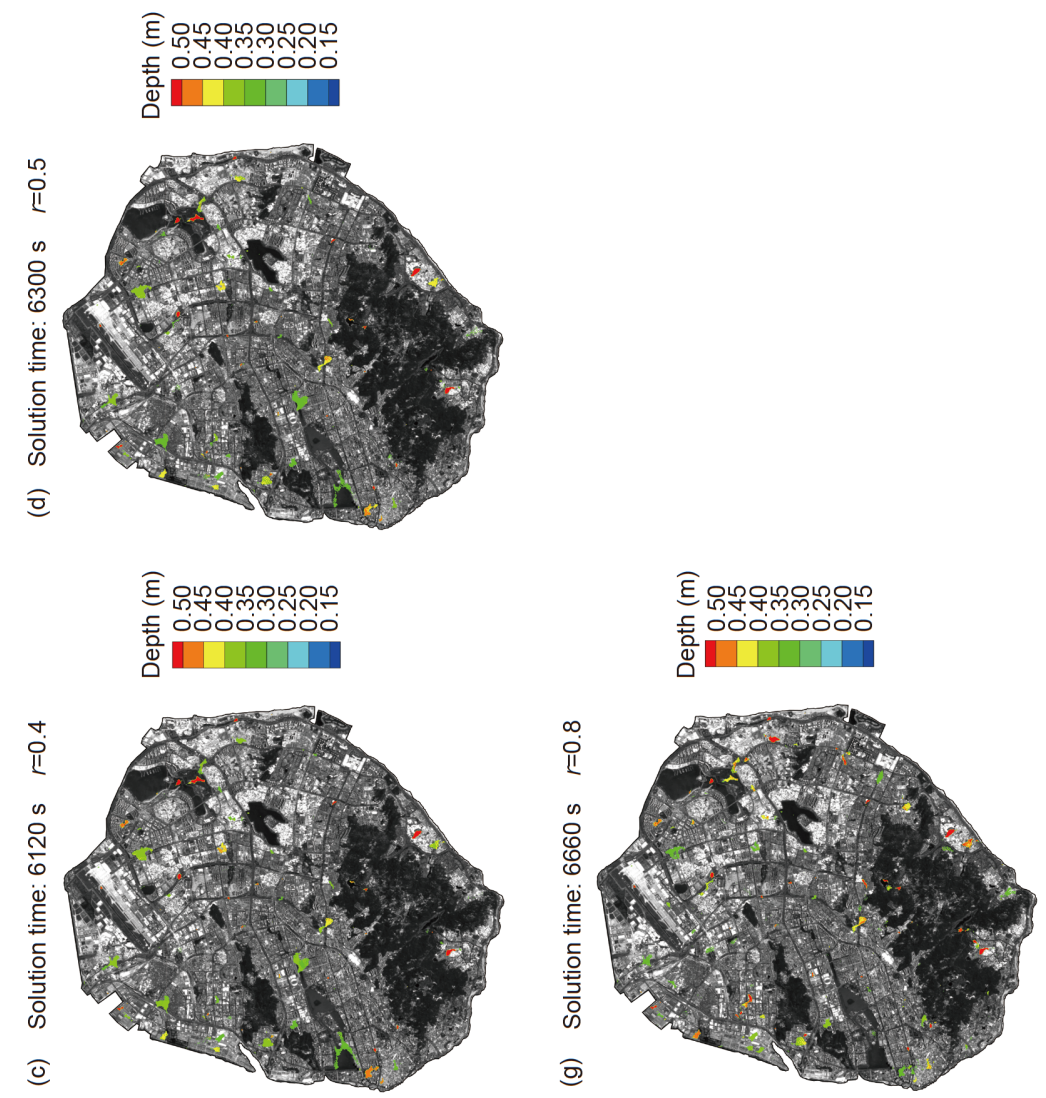

热
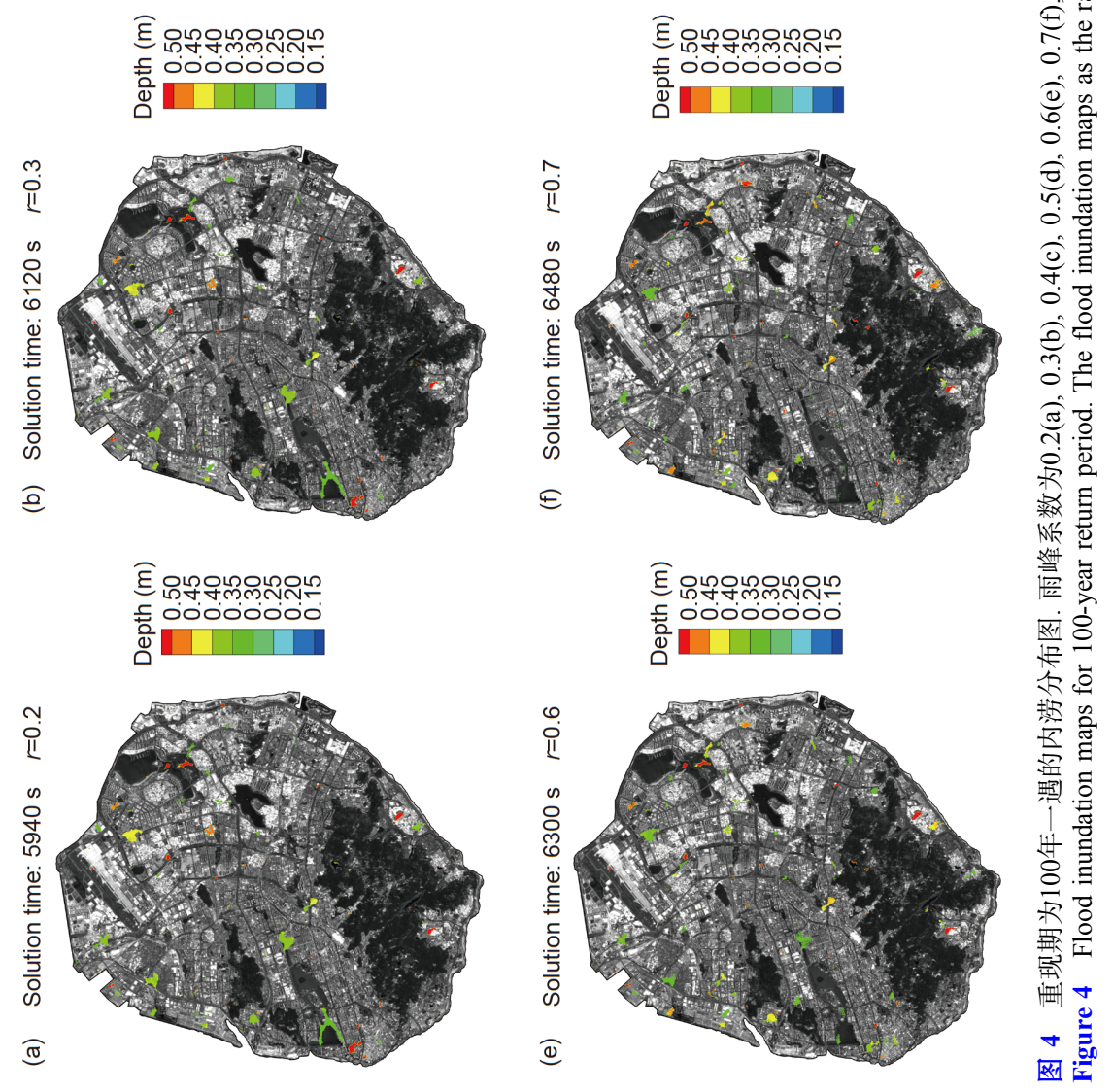

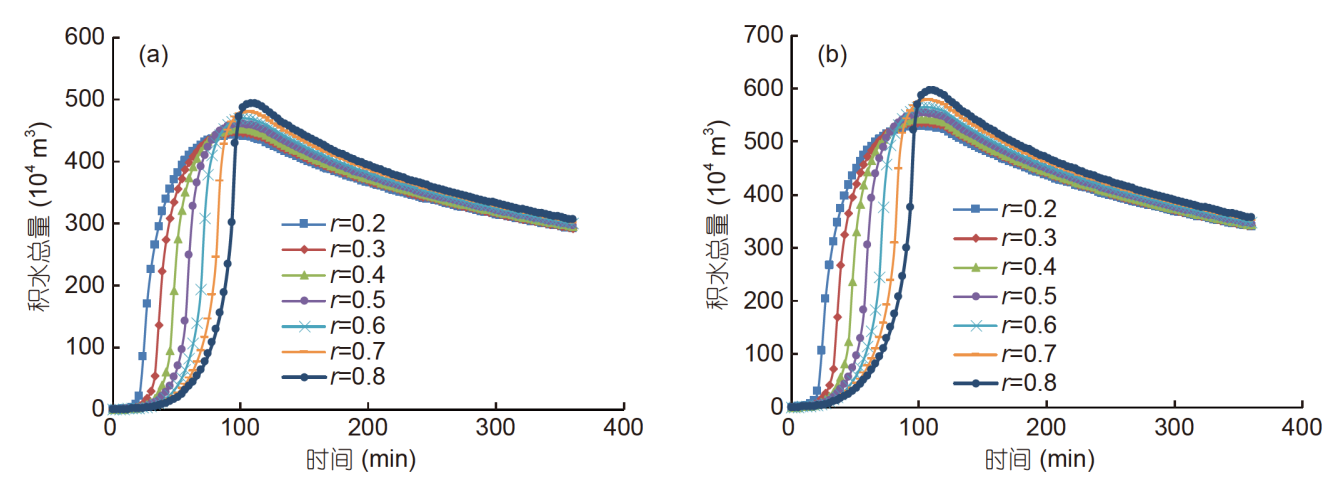

图 5 (网络版彩色)不同重现期设计暴雨过程积水总量变化. (a) 50年一遇; (b) 100年一遇

Figure 5 (Color online) The total inundation volume with different return periods. (a) 50-year return period; (b) 100-year return period

暴雨情况下积水总量的峰值, 见表 2.

从图4中可以看出, 在不同的雨峰系数下, 积水总 量达到峰值的时间是不同的, 并且峰值时刻的内涝积 水的分布范围和雨峰系数有较大的关系. 以位于厦门 岛西南方向的算筜湖区域为例, 在雨峰系数较小时, 算 筜湖周边是明显的内涝区; 在雨峰系数 $r=0.8$ 时, 算筜湖 周边的积水范围相比于 $r=0.2$ 时有明显的减少; 而位于 厦门岛东北方向的埭辽公园区域, 在不同的雨峰系数 下, 都是一个明显的内涝积水区域, 随着雨峰系数的不 同, 其积水深度也不同. 城市内涝积水充满了复杂性, 这不仅跟地形地貌特征有关, 也是由积水的漫流过程 和SCS模型的下渗过程来决定的.

以重现期 100 年为例, 雨峰系数 $r$ 从 0.2 增加到 0.3 , 积水总量增加了 6.4 万 $\mathrm{m}^{3}$, 增幅约 $1.21 \%$, 而雨峰系数从 0.7 增加到 0.8 , 积水总量增加了 16.9 万 $\mathrm{m}^{3}$, 增幅约 $2.92 \%$, 这表明在同一重现期下, 随着雨峰系数的增大, 积水总 量绝对值增大, 并且雨峰系数每变化一个单位, 积水总 量的相对增幅也在增大, 使得积水总量增大; 并且从图 5 中可以看出, 在不同重现期下, 随着重现期的增大, 积
水总量的绝对值增加得比较明显. 上述分析表明, 不同 重现期的设计降雨过程下城市内涝积水量变化规律基 本一致, 即重现期越大, 雨峰越靠后, 积水量越大.

\section{2 不同设计暴雨情景下的积水面积特征分析}

根据国家标准《GB51222-2017城镇内涝防治技术 规范》 ${ }^{[33]}$, 积水超过 $15 \mathrm{~cm}$ 视为发生内涝. 本文对积水深 度超过 $15 \mathrm{~cm}$ 的积水区域的积水面积进行了统计分析, 见图6所示.

从图6中可以看到, 积水面积的变化与雨峰系数有 较大的关系. 在降雨阶段, 由于考虑了下渗作用以及初 始地表没有积水的情景, 积水面积在刚开始都是低于 $15 \mathrm{~cm}$ 的; 在一段时间后积水面积开始增长, 并且因雨 峰系数的不同, 积水面积开始增长的时刻也是不同的, 雨峰系数越大, 积水面积开始增长的时刻越靠后, $r=0.2$ 时在约 $33 \mathrm{~min}$ 开始增长, $r=0.8$ 时在约 $84 \mathrm{~min}$ 开始 增长; 之后积水面积随降雨量的增加而不断增加, 在约 120 150 min时积水面积达到峰值. 在退水阶段, 积水面 积随时间逐渐消退, 由于仅仅考虑了SCS模型的下渗作

表 2 不同重现期和不同雨峰系数设计暴雨情景下积水总量的峰值 $\left(10^{4} \mathbf{~ m}^{3}\right)$

Table 2 The inundation volume peak values in different scenario simulations $\left(10^{4} \mathrm{~m}^{3}\right)$

\begin{tabular}{|c|c|c|c|c|c|c|}
\hline 雨峰系数 & $50 \mathrm{a}$ & 增幅 & 相对增幅(\%) & $100 \mathrm{a}$ & 增幅 & 相对增幅(\%) \\
\hline$r=0.2$ & 442.3 & & & 529.4 & & \\
\hline$r=0.3$ & 446.4 & 4.3 & 0.97 & 535.8 & 6.4 & 1.21 \\
\hline$r=0.4$ & 453.0 & 6.4 & 1.43 & 543.4 & 7.6 & 1.42 \\
\hline$r=0.5$ & 460.2 & 7.2 & 1.59 & 553.4 & 10.0 & 1.84 \\
\hline$r=0.6$ & 469.3 & 9.1 & 1.98 & 564.9 & 11.5 & 2.08 \\
\hline$r=0.7$ & 479.7 & 10.6 & 2.26 & 578.5 & 13.6 & 2.41 \\
\hline$r=0.8$ & 493.3 & 13.4 & 2.79 & 595.4 & 16.9 & 2.92 \\
\hline
\end{tabular}



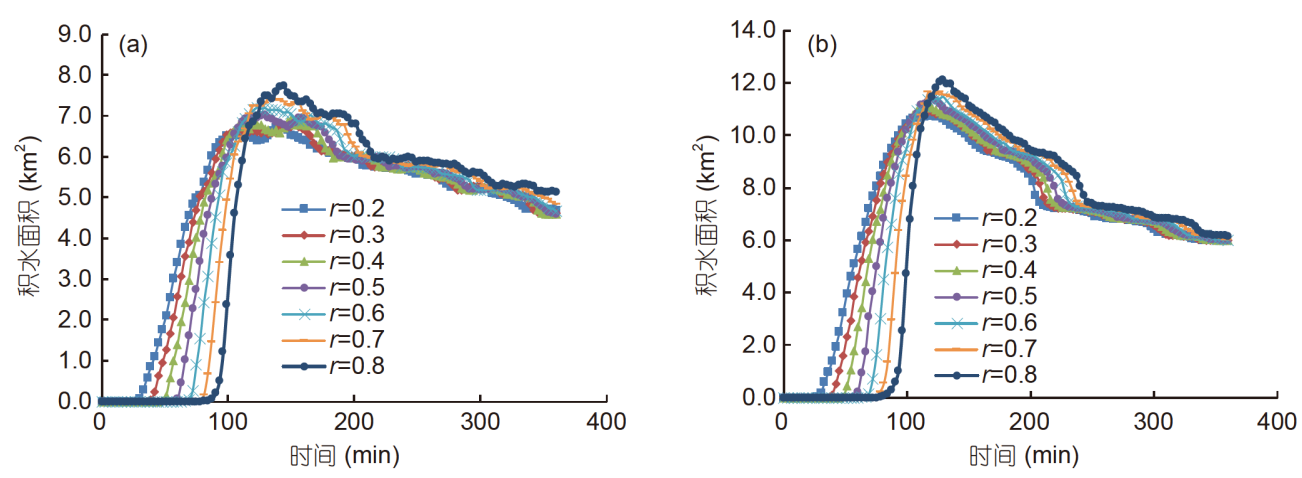

图 6 (网络版彩色)不同重现期设计暴雨情景下积水深度大于 $15 \mathrm{~cm}$ 的积水面积变化过程. (a) 50 年一遇; (b) 100年一遇

Figure 6 (Color online) The change process of inundation area that water depth greater than $15 \mathrm{~cm}$. (a) 50 -year return period; (b) 100-year return period

用而忽略了排水管网的作用，积水消退的途径只有下 渗和向岛外排出，因此在这个阶段存在与实际不相符 的情况. 通过对比不同重现期下积水总量的变化过程, 可以发现其变化规律是比较相似的.

根据相关文献资料对内涝风险等级划分的研究, 本文选取了 15 和 $30 \mathrm{~cm}$ 作为内涝风险等级的划分 ${ }^{[34,35]}$. 当积水深度超过 $15 \mathrm{~cm}$ 时，就会对道路的通行产生一定
的影响；当积水深度超过 $30 \mathrm{~cm}$ 时，就会对人员安全造 成危害并产生较为严重的经济损失. 因此, 对各场设计 暴雨情况下积水深度大于 15 和 $30 \mathrm{~cm}$ 的积水面积的峰 值进行了统计分析, 见表3和4及图7.

对于积水深度大于 $15 \mathrm{~cm}$ 的积水面积，在不同重现 期下, 积水面积随雨峰系数的增大而增大, 并且相对增 幅随着雨峰系数的增大而整体上逐渐增大, 当雨峰系数

\section{表 3 各场设计暴雨过程积水深度大于 $15 \mathrm{~cm}$ 的积水面积峰值 $\left(\mathrm{km}^{2}\right)$}

Table 3 The peak values of inundation area that water depth greater than $15 \mathrm{~cm}^{(\mathrm{km})}$

\begin{tabular}{|c|c|c|c|c|c|c|}
\hline 雨峰系数 & $50 \mathrm{a}$ & 增幅 & 相对增幅(\%) & $100 \mathrm{a}$ & 增幅 & 相对增幅(\%) \\
\hline$r=0.2$ & 6.73 & & & 10.73 & & \\
\hline$r=0.3$ & 6.77 & 0.04 & 0.53 & 10.91 & 0.18 & 1.67 \\
\hline$r=0.4$ & 6.89 & 0.12 & 1.83 & 11.11 & 0.20 & 1.81 \\
\hline$r=0.5$ & 7.01 & 0.12 & 1.74 & 11.41 & 0.30 & 2.66 \\
\hline$r=0.6$ & 7.20 & 0.19 & 2.66 & 11.46 & 0.05 & 0.47 \\
\hline$r=0.7$ & 7.43 & 0.23 & 3.21 & 11.67 & 0.21 & 1.84 \\
\hline$r=0.8$ & 7.73 & 0.30 & 4.04 & 12.14 & 0.46 & 3.95 \\
\hline
\end{tabular}

表 4 各场设计暴雨过程积水深度大于 $30 \mathrm{~cm}$ 的积水面积峰值 $\left(\mathrm{km}^{2}\right)$

Table 4 The peak values of inundation area that water depth greater than $30 \mathrm{~cm}^{\left(\mathrm{km}^{2}\right)}$

\begin{tabular}{|c|c|c|c|c|c|c|}
\hline 雨峰系数 & $50 \mathrm{a}$ & 增幅 & 相对增幅(\%) & $100 \mathrm{a}$ & 增幅 & 相对增幅(\%) \\
\hline$r=0.2$ & 1.68 & & & 2.42 & & \\
\hline$r=0.3$ & 1.67 & -0.01 & -0.59 & 2.43 & 0.01 & 0.41 \\
\hline$r=0.4$ & 1.68 & 0 & 0.23 & 2.36 & -0.07 & -2.88 \\
\hline$r=0.5$ & 1.68 & 0 & 0.10 & 2.31 & -0.05 & -2.12 \\
\hline$r=0.6$ & 1.68 & 0 & 0.11 & 2.32 & 0.01 & 0.43 \\
\hline$r=0.7$ & 1.68 & 0 & -0.05 & 2.36 & 0.04 & 1.72 \\
\hline$r=0.8$ & 1.68 & 0 & -0.08 & 2.43 & 0.07 & 2.97 \\
\hline
\end{tabular}



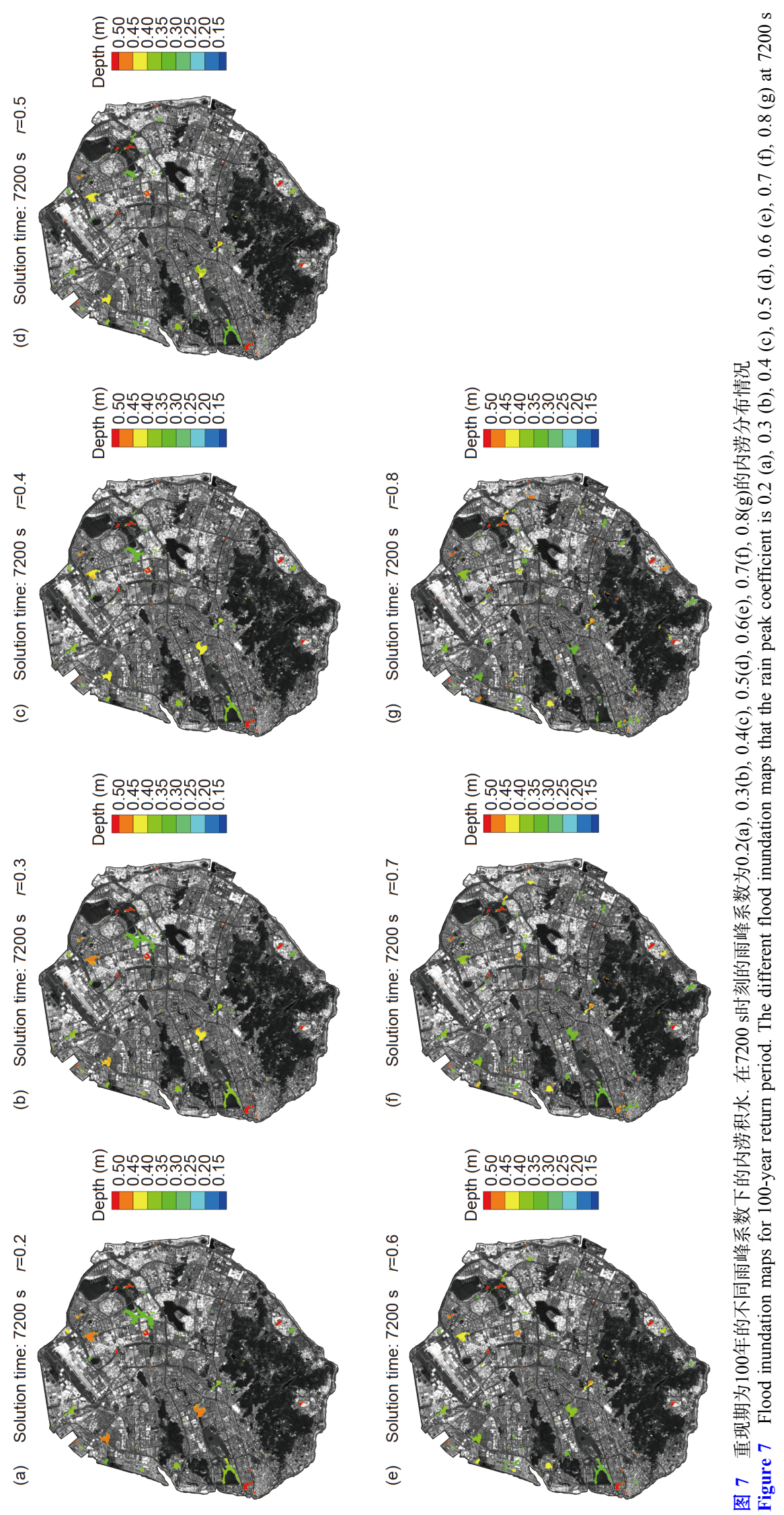
$r=0.8$ 时积水面积达到最大值. 对于积水深度大于 $30 \mathrm{~cm}$ 的积水面积, 在不同重现期下, 积水面积与雨峰系数的 关系不明显, 不同雨峰系数引起的积水面积的变化有增 有减. 通过表 3 和 4 的分析, 可以知道, 对于不同的内涝风 险等级划分, 雨峰系数的变化带来的影响是不同的. 对 于 $15 \mathrm{~cm}$ 积水深度, 雨峰系数越大, 积水面积越大; 对于 $30 \mathrm{~cm}$ 积水深度, 雨峰系数没有明显的影响.

从上述分析可知，在做城市内涝的预警预报和抢 险救灾时，需要对不同风险等级的积水考虑不同的措 施. 对 $15 \mathrm{~cm}$ 积水深度的积水, 要着重注意峰现时间较 晚的降雨, 对于 $30 \mathrm{~cm}$ 积水深度的降雨, 对不同峰现时 间的降雨都要关注.

\section{3 不同设计暴雨情景下的峰现时间特征分析}

由于降雨需要经过产流和汇流的过程才会形成内 涝积水, 并且这个过程伴随着一系列复杂的水文水动 力学过程, 所以积水总量峰值出现的时刻相比于降雨 峰值出现的时刻总是有一定的延迟. 本文将内涝峰值 (积水总量峰值)出现时刻相比于暴雨峰值出现时刻的 时间差称为迟滞时间. 通过对这个迟滞效应的分析研 究可以对内涝灾情的预警预报提供有效的科学依据. 图7以重现期为 100 年为例, 绘制了同一时刻不同雨峰 系数下的积水内涝分布图.

通过对14场设计暴雨的模拟结果的统计分析, 各 场设计暴雨的内涝积水总量的迟滞时间统计结果见表 5所示.

从表 5 中可见: 在同样的重现期下, 雨峰系数越大, 迟滞时间越短, 并且在不同的重现期下, 这个规律都存 在. 在雨峰系数 $r=0.8$ 时, 迟滞时间只有 12 或 $15 \mathrm{~min}$, 而 $r=0.2$ 时, 迟滞时间长达 69 或 $75 \mathrm{~min}$. 在进行城市内涝的 防治管理时, 要特别注意降雨的雨峰特征, 针对不同情 景, 进行有效的预警预报.

\section{4 排水管网概化的讨论}

城市内涝的数值模拟, 其最主要的特征在于城市 地表和地下的双层耦合结构. 目前主要的数值模拟方 法包括两种: (1) 水动力学方法, 即地下一维管道水动 力学模型和地表二维水动力学模型; (2) 水文水动力学 方法, 即地下一维管道水动力学模型和地表二维产汇 流水文学方法.

地下排水管网是城市内涝模拟的重要组成部分, 但是由于排水管网数据的难以收集、分布复杂等原因,
表 5 各场设计暴雨内涝积水总量迟滞时间( min)

Table 5 The hysteresis of inundation volume in different scenario simulations ( $\mathrm{min})$

\begin{tabular}{ccc}
\hline 迟滞时间 & $50 \mathrm{a}$ & $100 \mathrm{a}$ \\
\hline$r=0.2$ & 69 & 75 \\
$r=0.3$ & 60 & 66 \\
$r=0.4$ & 51 & 54 \\
$r=0.5$ & 39 & 45 \\
$r=0.6$ & 30 & 33 \\
$r=0.7$ & 21 & 24 \\
$r=0.8$ & 12 & 15 \\
\hline
\end{tabular}

在实际建模过程中通常采用概化方法进行处理. 已有 文献[35 38]对管网概化的基本方法和基本原则做了介 绍. 本文建立的二维水动力学模型对排水管网的处理 采用了通用的概化方法，即根据相应的建设设计规范， 将管网的排水作用视为等效下渗, 通过在降雨数据中 扣除一定的水量, 以一个静态值作为管网的排水量, 忽 略管网实际的水动力学过程. 但这种概化方法存在系 统性误差，即：在降水发生的时间内，管网近似处于充 满状态, 忽略了管网的排水计算, 而是采用等效的方法 对管网的排水作用进行考虑; 但在降水结束后管网持 续的排水作用由于降水的停止而无法扣除, 实际排水 功能没有考虑. 这种概化方法在欧洲各国使用比较广 泛，这主要是因为欧洲各国重点关注城市洪涝淹没发 生发展的前半段过程及其最大淹没范围和淹没水深 (这是确定洪涝风险的两个重要参数), 而这种处理方法 在洪涝淹没的发生发展阶段误差较小, 能够满足精度 要求. 以本文所建立的厦门岛二维水动力学模型为例, 通过对图 5 和 6 的分析, 在淹没期间, 即降雨强度大于排 水强度，采用等效的方法计算了排水管网的排水作用, 积水总量和积水面积的计算结果比较可靠; 在退水阶 段, 由于不考虑管网作用, 积水消退仅依赖于下渗和向 岛外自由出流，积水总量和积水面积的消退过程就会 比较缓慢, 计算结果的可靠性较低. 在单纯的二维水动 力学模拟中, 为了将退水阶段管网作用进行适当的考 虑, 可以采取将模型的下渗值进行合理调整的方法, 增 大下渗率，在本文中是通过调整 $\mathrm{CN}$ 参数来实现这一 目的.

综上所述, 在缺乏管网数据的情况下, 对排水管网 进行适当的并且科学的概化很有必要, 在概化的过程中 不仅要考虑排水管网的实际排水能力, 还需要将其细分 
为降雨阶段和积水消退阶段进行考虑. 如果要更为精细 的模拟积水淹没和消退过程, 就需要建立一二维耦合模 型, 综合考虑地下排水管网和地上地表产汇流过程.

\section{4 结论}

本文基于TELEMAC-2D模型构建了厦门岛的城市 内涝模型，对14场设计暴雨情景下的厦门岛的积水总 量、淹没面积和迟滞时间进行了模拟计算，初步揭示 了不同设计暴雨下的厦门岛城市内涝的基本规律, 主 要结论如下:

(1) 积水总量受雨峰系数的影响较大. 雨峰系数越 大, 积水总量的峰值越大, 并且每变化一个单位的雨峰 系数, 积水总量的增幅和相对增幅都在增大.

(2) 积水面积的变化不仅与雨峰系数有关, 也与重 现期有关. 在同一重现期下, 雨峰系数越大, 积水面积 越大.

（3）不同内涝风险等级的积水面积的变化也受到
雨峰系数的影响，对于积水深度大于 $15 \mathrm{~cm}$ 的积水面 积, 不同雨峰系数对积水面积的影响比较明显, 积水面 积随着雨峰系数的增大而增大; 而积水深度大于 $30 \mathrm{~cm}$ 的积水面积, 雨峰系数对积水面积没有明显的影响.

（4）在同样的重现期下，雨峰系数越大，迟滞时间 越短, 并且在不同的重现期下, 这个规律都存在.

本研究初步分析了不同重现期和雨峰系数下的厦 门岛城市内涝特征，对于该地区科学防治城市内涝有 一定的参考意义. 本次数值模拟中, 地形数据的分辨率 较低, 曼宁系数和 $\mathrm{CN}$ 值等参数参考了临近区域的相关 文献，尽可能贴近实际情况. 此外，由于缺乏相关的城 市排水管网基本资料，在本次模拟中将排水管网的作 用进行概化, 特别是在降水结束后, 管网的排水作用无 法在模型中体现，直接导致模拟后期的结果与实际情 况偏差较大, 这是单纯二维城市洪涝模型的通病, 为了 更加精确地模拟城市洪涝全过程的淹没和退水特征, 需要构建一二维耦合的城市洪涝模型.

\section{参考文献}

1 Zhang J Y, Song X M, Wang G Q, et al. Development and challenges of urban Hydrology in a changing environment I: Hydrological response to urbanization (in Chinese). Adv Water Sci, 2014, 25: 594-605 [张建云, 宋晓猛, 王国庆, 等. 变化环境下城市水文学的发展与挑战一-I. 城市 水文效应. 水科学进展, 2014, 25: 594-605]

2 Song X M, Zhang J Y, Wang G Q, et al. Development and challenges of urban hydrology in a changing environment II: Urban stormwater modeling and management (in Chinese). Adv Water Sci, 2014, 25: 752-764 [宋晓猛, 张建云, 王国庆, 等. 变化环境下城市水文学的发展与挑战一一II. 城市雨洪模拟与管理. 水科学进展, 2014, 25: 752-764]

3 Wang H, Mei C, Liu J H. Systematic construction pattern of the sponge city (in Chinese). J Hydraul Eng, 2018, 48: 1009-1014 [王浩, 梅超, 刘家 宏. 海绵城市系统构建模式. 水利学报, 2018, 48: 1009-1014]

4 Zhang J Y, Wang Y T, He R M, et al. Discussion on the urban flood and waterlogging and causes analysis in China (in Chinese). Adv Water Sci, 2016, 27: 485-491 [张建云, 王银堂, 贺瑞敏, 等. 中国城市洪涝问题及成因分析. 水科学进展, 2016, 27: 485-491]

5 Ren M F, Xu Z X, Huang Z Q, et al. Simulations of rainstorm waterlogging processes around Lianghua bridge in Beijing (in Chinese). J Hydroelectr Eng, 2017, 36: 10-18 [任梅芳, 徐宗学, 黄子千, 等. 北京莲花桥区域暴雨积水模拟研究. 水力发电学报, 2017, 36: 10-18]

6 Xiang J H, Wang L. Discussion on coping with urban waterlogging disaster in WuHan, Hubei Province (in Chinese). Chin Flood Drought Manage, 2013, 23: 24-27 [项久华, 王亮. 湖北武汉市应对城市内涝灾害探讨. 中国防汛抗旱, 2013, 23: 24-27]

7 Ma J Y. Analysis on the causes of waterlogging formation in Shenzhen City and study on waterlogging treatment (in Chinese). Water Resour Hydr Eng, 2015, 46: 105-111 [马晋毅. 深圳市内涝形成原因分析与治涝对策研究. 水利水电技术, 2015, 46: 105-111]

8 Shi S, Lin K P, Chen R R, et al. Xiamen city analysis and countermeasure of water logging (in Chinese). J Meteorol Res Appl, 2014, 35: 44-48 [施 斯, 林开平, 陈荣让, 等. 厦门市城市内涝成因研究与对策分析. 气象研究与应用, 2014, 35: 44-48]

9 Zhou H, Liu J, Gao C, et al. Analysis of current situation and problems of urban waterlogging control in China (in Chinese). J Catastrophol, 2018, 33: 147-151 [周宏, 刘俊, 高成, 等. 我国城市内涝防治现状及问题分析. 灾害学, 2018, 33: 147-151]

10 Sivapalan M, Savenije H H G, Blöschl G. Socio-hydrology: A new science of people and water. Hydrol Process, 2012, 26: 1270-1276

11 Liu J H, Wang H, Gao X R, et al. Review on urban hydrology (in Chinese). Chin Sci Bull, 2014, 59: 3581-3590 [刘家宏, 王浩, 高学䜭, 等. 城市 水文学研究综述. 科学通报, 2014, 36: 3581-3590]

12 Teng J, Jakeman A J, Vaze J, et al. Flood inundation modelling: A review of methods, recent advances and uncertainty analysis. Environ Model Software, 2017, 90: 201-216

13 UN. World Urbanization Prospects: The 2011revision. New York: UN-HABITAT, 2018

14 National Bureau of Statistic. 2017 Statistical Bulletin on National Economic and Social Development of the People's Republic of China (in Chinese). 2018 [中华人民共和国国家统计局. 中华人民共和国2017年国民经济和社会发展统计公报. 2018]

15 Seenath A, Wilson M, Miller K. Hydrodynamic versus GIS modelling for coastal flood vulnerability assessment: Which is better for guiding coastal management? Ocean Coast Manage, 2016, 120: 99-109 
16 Bach P M, Rauch W, Mikkelsen P S, et al. A critical review of integrated urban water modelling-Urban drainage and beyond. Environ Model Software, 2014, 54: 88-107

17 Huang G R, Wang X, Huang W. Simulation of rainstorm water logging in urban area based on InfoWorks ICM model (in Chinese). Water Resour Power, 2017, 35: 66-70 [黄国如, 王欣, 黄维. 基于InfoWorks ICM模型的城市暴雨内涝模拟. 水电能源科学, 2017, 35: 66-70]

18 Chen X, Ji P, Wu Y, et al. Coupling simulation of overland flooding and underground network drainage in a coastal nuclear power plant. Nucl Eng Des, 2017, 325: 129-134

19 Shi S. The island city to cope with meteorological disasters vulnerability evaluation analysis and countermeasures-A case study in Xiamen City (in Chinese). Master Dissertation. Nanning: Guangxi Teachers Education University, 2015 [施斯. 海岛型城市应对气象灾害的城市脆弱性评价分析 与对策建议——厦门市为例. 硕士学位论文. 南宁: 广西师范学院, 2015]

20 Fujian Climate Center. Xiamen Urban Rainstorm Intensity Formula Revision and Design Rain Type Research Technical Report (in Chinese). 2016 [福建省气候中心. 厦门市城市暴雨强度公式修编和设计雨型研究技术报告. 2016]

21 Ministry of Housing and Urban-Rural Development of the People's Republic of China, General Administration of Quality Supervision Inspection and Quarantine of the People's Republic of China. Code for design of outdoor wastewater engineering (2014) (in Chinese): GB50014-2006. Beijing: China Planning Press, 2014 [中华人民共和国住房和城乡建设部, 中华人民共和国质量监督检验检疫总局. 室外排水设计规范(2014 年版): GB50014-2006. 北京: 中华计划出版社, 2014]

22 Hou J M, Guo K H, Wang Z L, et al. Numerical simulation of design storm pattern effects on urban flood (in Chinese). Adv Water Sci, 2017, 28: 820-828 [侯精明, 郭凯华, 王志力, 等. 设计暴雨雨型对城市内涝影响数值模拟. 水科学进展, 2017, 28: 820-828]

23 United States Department of Agriculture. Urban Hydrology for Small Watersheds. Technical Release 55 (TR-55). 2nd ed. Natural Resources Conservation Service, Conservation Engineering Division. 1986

24 Huang J L, Lin J, Du P F. Parameter uncertainty analysis for urban rainfall runoff modelling (in Chinese). Environ Sci, 2012, 33: $2224-2234$ [黄金 良, 林杰, 杜鹏飞. 城市降雨径流模拟的参数不确定性分析. 环境科学, 2012, 33: 2224-2234]

25 Tang Z Q. Flood simulation and visualization of flood elements based on GIS (in Chinese). Master Dissertation. Wuhan: Huazhong University of Science and Technology, 2013 [汤中倩. 基于GIS的洪水仿真及洪水要素可视化. 硕士学位论文. 武汉: 华中科技大学, 2013]

26 Galland J C, Goutal N, Hervouet J M. TELEMAC: A new numerical model for solving shallow water equations. Adv Water Resour, 1991, 14: 138148

27 Brière C, Abadie S, Bretel P, et al. Assessment of TELEMAC system performances, a hydrodynamic case study of Anglet, France. Coast Eng, 2007, 54: 345-356

28 Hervouet J M. Hydrodynamics of Free Surface Flows: Modelling with the Finite Element Method. Chichester (England): John Wiley \& Sons, 2007. 83-133

29 Li R K, Zhu A X, Chen L J, et al. Effects of soil parameters in SCS-CN runoff model (in Chinese). J Nat Resour, 2013, 28: 1778-1787 [李润奎, 朱 阿兴, 陈腊娇, 等. SCS-CN模型中土壤参数的作用机制研究. 自然资源学报, 2013, 28: 1778-1787]

30 Pierre L L. Implementation of a rainfall-runoff model in TELEMAC-2D. XXIIIrd TELEMAC-MASCARET User Conference. 2016. 13-20

31 Lin M X. Study on rainstorm waterlogging disaster risk base on the cintruction of sponge city: A case study of Xiamen (in Chinese). Master Dissertation. Xi'an: Xi'an University of Science and technology, 2017 [林美霞. 基于海绵城市建设的厦门城市暴雨内涝灾害风险研究. 硕士学 位论文. 西安: 西安科技大学, 2017]

32 Xiao Y H. Relationship between landscape pattern and surface runoff based on GIS in Fuzhou (in Chinese). Master Dissertation. Fuzhou: Fujian Agriculture and Forestry University, 2017 [肖以恒. 基于GIS支持下福州城区景观格局与地表径流量关系研究. 硕士学位论文. 福州: 福建农林大学, 2017]

33 Ministry of Housing and Urban-Rural Development of the People's Republic of China. Technical Specification for Prevention and Control of Urban Waterlogging (in Chinese). Beijing: China Planning Press, 2017 [中华人民共和国住房和城乡建设部. 城镇内涝防治技术规范: GB51222-2017. 北京: 中国计划出版社, 2017]

34 Dai J J, Liu Z X, Lu S J. Study on the risk assessment of urban waterlogging based on numerical simulation: Taking Suzhou urban central area as an example (in Chinese). Water Resour Plann Design, 2015, 6: 40-43 [戴晶晶, 刘增贤, 陆沈钧. 基于数值模拟的城市内涝风险评估研究一一以苏 州市城市中心区为例. 水利规划与设计, 2015, 6: 40-43]

35 Wang F Y, Ying G D, Jin L P, et al. Research and Analysis on the model of urban water logging in Linhai City (in Chinese). Bull Sci Tech, 2018, 4: 67-71 [王峰波, 应国德, 金凌鹏, 等. 临海城市内涝模型研究与分析. 科技通报, 2018, 4: 67-71]

36 Li S H. Analysis flood risk and study waterlogging simulation in Zhengzhou (in Chinese). Master Dissertation. Zhengzhou: Zhengzhou University, 2016 [李世豪. 郑州市区洪涝风险分析及内涝积水模拟研究. 硕士学位论文. 郑州: 郑州大学, 2016]

37 Lü H, Ni G H, Tian F Q. Impacts of drainage pipe network complexity on urban stormwater modeling (in Chinese). J Hydroelectr Eng, 2018, 37: 97-106 [吕恒, 倪广恒, 田富强. 排水管网结构概化对城市暴雨洪水模拟的影响. 水力发电学报, 2018, 37: 97-106]

38 Liang Q, Smith L S. A high-performance integrated hydrodynamic modelling system for urban flood simulations. J Hydroinform, 2015, 17: 518-533 


\title{
Urban flood analysis for different design storm hyetographs in Xiamen Island based on TELEMAC-2D
}

\author{
Jiahong Liu ${ }^{1,3^{*}}$, Zejin $\mathrm{Li}^{1}$, Chao Mei ${ }^{1}$, Kaibo Wang ${ }^{1}$ \& Guannan Zhou ${ }^{2}$ \\ ${ }^{1}$ China Institute of Water Resources and Hydropower Research, State Key Laboratory of Simulation and Regulation of Water Cycle in River Basin, \\ Beijing 100038, China; \\ ${ }^{2}$ China Railway Construction Bridge Engineering Group Co., Ltd., Tianjin 300300, China; \\ ${ }^{3}$ Engineering and Technology Research Center for Water resources and Hydroecology of the Ministry of Water Resources, Beijing 100044, China \\ * Correspinding author, E-mail: liujh@iwhr.com
}

Urban flood is more serious and more frequent with the rapid urbanization in recent years. Numerical simulation is one of the important means to study urban flood, which is widely used in analyzing the characteristics of urban waterlogging. In this paper, a numerical model had been established to simulate the urban waterlogging in Xiamen Island based on TELEMAC-2D software. TELEMAC-2D is one of the two-dimensional hydrodynamic models, which developed by the French National Hydraulic and Environment Laboratory. The finite element method (FEM) was used to solve the NavierStokes equations, and Soil Conservation Service Curve Number (SCS-CN) method was used to simulate rainfall-runoff processes. The urban flooding processes under different design storm hyetographs were simulated, with return periods of $50 / 100$ a and rain peak coefficients of $0.2,0.3,0.4,0.5,0.6,0.7$, and 0.8 , respectively. The contrastive analyses had been done for 14 simulation scenarios, which consisted the terms of the total water accumulation, inundation area and hysteresis of inundation. The results show that the total water accumulation is greatly affected by the rain peak coefficient, and the higher the rain peak coefficient is, the greater the peak value of the total water accumulation is. The inundation area is also affected by the rain peak coefficient. Under the same return period of rainfall, the inundation area increases with the increase of rain peak coefficient. The inundation area of different depth $(>15 \mathrm{~cm},>30 \mathrm{~cm})$ varies with the rain peak coefficient. For the flooded area with water depth greater than $15 \mathrm{~cm}$, the area increases with the increase of rain peak coefficient, while for that greater than $30 \mathrm{~cm}$, the effect of rain peak coefficient is not obvious. Under the same return period of rainfall, the higher the rain peak coefficient is, the shorter the hysteresis of inundation is. These conclusions made a preliminary revelation of the characteristic of urban flooding processes in urban area, which could provide basic support for urban flood forecast and regulation. For the lack of detailed drainage pipe network, a simplified method was used in modelling the drainage effect of pipe network, which deducted the designed rainfall of drainage pipe network from the total rainfall, and then input it to the simulation model. The simplified method is basically correct during the rain events, while there are systematic errors in the receding stage after the rainfall stops. As the rainfall is zero after rain stops, there is no way to deduct the designed rainfall of drainage pipe network, so the drainage function of the pipe network can't be reflected after rain stops. That is why the simulated receding time of the simplified method is longer than the actual result. Since most of the waterlogging simulation focuses on the occurrence and expansion of inundating during rainfall, the simplified method is widely used in European and other western countries. If the process of inundation is a focus for concern, the simplified method is not applicable, under which the suggested model needs to be improved to simulate the drainage process of the pipe-network in detail.

urban flood, TELEMAC-2D hydrodynamic model, inundation simulation, urbanization, Xiamen Island doi: $10.1360 / \mathrm{N} 972018-01180$ 